\title{
Implementing Clinical Preventive Medicine: Time to Fish or Cut Bait
}

\author{
Paul S. Frame, MD
}

Numerous recent studies have documented that primary care physicians routinely fail to offer proven preventive procedures to many of their patients on a routine basis. In this issue of The fournal, Ruffin and colleagues. ${ }^{1}$ report results from a study of cancer screening in 24 community-based primary care practices. Their results again confirm that rates of offering cancer-screening tests are low.

In part, the poor results could be the result of including controversial tests or tests of unproved benefit, which practitioners might reasonably and rationally exclude from their preventive protocols. The authors appear to have used the American Cancer Society guidelines as their standard and studied tests such as the digital rectal examination, prostate-specific antigen, and breast self-examination, which are of unproved benefit. They also looked at breast cancer screening for women younger the age of 50 years and used an annual mammography interval for women older than 50 years, both of which are controversial. ${ }^{2}$

Even if we look only at those tests and screening intervals that are uniformly recommended, however, the results leave much to be desired. Only $63.8 \%$ of women with a cervix had a Papanicolaou smear within 3 years, $55 \%$ of women older than 50 years had had a mammogram within 2 years, and no more than $46 \%$ of persons older than 50 years had either had flexible sigmoidoscopy within 5 years or fecal occult blood testing within the past year.

The results are probably optimistic and overstate the amount of cancer screening going on in the general community. The study only looked at charts of active patients, defined as having at least 2 visits to the practice in the past 2 years. Inactive patients are likely to be less compliant with cancer screening. Eighty-eight practices were invited to

Submitted 4 November 1999.

From a private practice, Cohocton, and the Department of Family Medicine, University of Rochester School of Medicine and Dentistry, Rochester, NY. Address reprint requests to Paul S. Frame, MD, Tri-County Family Medicine, 25 Park Avenue, Cohocton, NY 14826. participate in the study. Thirty-eight practices that were eligible refused to participate, leaving 24 practices in the study. The practices that refused to participate are not likely to be ones placing a high priority on prevention. Thus, actual populationwide compliance with proven cancer screening tests is probably less than the results reported by Ruffin and colleagues.

What constitutes good rates of provision of cancer prevention services? Community-based studies of physician-prompting and reminder systems have shown mixed results, some of which are no better than the results reported by Ruffin et al. McPhee et $\mathrm{al}^{3}{ }^{3}$ in an older study of computer-based physician reminders, reported $40.4 \%$ of women had a mammogram in the past year, $44.8 \%$ had a Papanicolaou smear in the past 3 years, and $50.4 \%$ of persons older than 50 years had a fecal occult blood test within the past year. Frame et $a .^{4}$ reported a study using a computer-based provider and patient reminder system. They found that $62 \%$ of women had a Papanicolaou smear in the past 2 years, $55 \%$ of women older than 50 years had a mammogram within 2 years, and $58 \%$ of persons older than 50 years had a fecal occult blood test within the past year.

Cancer screening rates reported to the National Committee on Quality Assurance (NCQA) by participating health maintenance organizations are higher. The national average compliance of women aged 21 to 64 years having had a Papanicolaou smear within 3 years as reported to the NCQA is $69.8 \%$, whereas the 90 th percentile is $81 \%$. The national average compliance of women aged 52 to 64 years having a mammogram within 2 years reported to the NCQA was $72.2 \%$, whereas the 90th percentile was $81 \%$ (Black E, Blue-Cross BlueShield of the Rochester area, personal communication, 1999). The accuracy of these data is not known. A recent analysis of Medicare data found that $55.4 \%$ of women aged between 65 and 69 years had had a mammogram within the past 2 years. ${ }^{5}$ In this study, $78 \%$ of women whose primary care 
provider was a gynecologist, $67 \%$ of women whose primary care provider was an internist, and $58 \%$ of women whose primary care provider was a family physician had a mammogram in the past 2 years. In an imperfect world $100 \%$ compliance with cancer screening tests is not likely to be achieved. A more reasonable and realistically achievable goal is to have compliance rates greater than $75 \%$.

Preventive services will not be consistently delivered to a great majority of patients until practices have institutionalized systems for ensuring the offering of preventive care. Such systems must include the following components:

1. A written practice preventive health protocol

2. Specific delegation of responsibility for doing preventive procedures

3. Involvement of patients

4. A system of periodic audits to evaluate whether goals are being met

5. Provider (and possibly patient) reinforcements for compliance with the health maintenance protocols

Institutionalizing prevention costs money, either directly or through the time and effort of providers and staff. Most practices have not given preventive services a high enough priority to warrant the necessary expenditure of scarce resources. The most telling statement in the study by Ruffin and colleagues ${ }^{1}$ was that "no practice had written or documented policies or protocols for cancer screening." If practices have no written preventive health protocol, it is unlikely they have developed a system for delivering preventive services.

That patients visiting for an annual health examination had the highest rates of compliance with cancer screening is not surprising. In a sense the annual examination is a system, albeit a crude one, for delivering preventive services. Any system is likely to be better than no system at all. It would be interesting to know whether these annual health examinations are patient initiated or provider initiated. It is possible that a subset of patients is demanding preventive services from their providers by requesting health maintenance examinations.

The discipline of family medicine claims to specialize in people. This claim rings hollow if we do not consistently offer preventive services to all our patients. All practices should have a written health maintenance protocol and a system for implementing it. Residency programs should be role models for this process. Documentation of a system for delivering preventive services should be a requirement for accreditation of family practice residency programs. If family physicians do not become the leaders in practicing prevention, we will loose credibility with specialist colleagues, patients, and government agencies. We will have no one to blame but ourselves.

\section{References}

1. Ruffin MT, Gorenflo DW, Woodman B. Predictors of screening for breast, cervical, colorectal, and prostatic cancer among community-based primary care practices. J Am Board Fam Pract 2000;13: 000-

2. Kerlikowske K, Grady D, Rubin SM, Sandrock C, Ernster VL. Efficacy of screening mammography: a meta-analysis. JAMA 1995;273:149-54.

3. McPhee SJ, Bird JA, Fordham D, Rodnick JE, Osborn EH. Promoting cancer prevention activities by primary care physicians. Results of a randomized, controlled trial. JAMA 1991;266:538-44.

4. Frame PS, Zimmer JG, Werth PL, Hall WJ, Eberly SW. Computer-based vs manual health maintenance tracking. A controlled trial. Arch Fam Med 1994;3: 581-8.

5. Finison KS, Wellins CA, Wennberg DE, Lucas FL. Screening mammography rates by specialty of the usual care physician. Eff Clin Pract 1999;2:120-5. 